\title{
Time Resolved Infrared Analysis of Droplet Impacts onto Heated Surfaces Under Extreme Wetting Scenarios
}

\author{
P. Pontes, E. Teodori ${ }^{1}$, A. S. Moita*1, A. L. N. Moreira ${ }^{1}$ \\ ${ }^{1} \mathrm{IN}+$ - Center for Innovation, Technology and Policy Research, Instituto Superior Técnico, \\ Universidade de Lisboa, Lisbon, Portugal \\ ${ }^{*}$ Corresponding author: anamoita@tecnico.ulisboa.pt
}

\begin{abstract}
The present paper explores the use of time resolved infrared IR thermography combined with high-speed imaging to describe the liquid-surface interfacial heat transfer phenomena occurring at droplet/wall interactions. Custom made calibration and post-processing methods are proposed and discussed. The results show that the methodology proposed captures very well particular details on droplet dynamics and heat transfer, allowing to identify air bubble trapping at the impact region as well as the temperature variations at the formation of the rim. Furthermore, the calibration proposed here allowed amending some physically incorrect results that were often obtained with the IR camera's default calibration. The combined analysis of droplet dynamics (e.g. the spreading factor) with the radial temperature profiles, heat flux and cooling effectiveness computation allowed establishing qualitative and quantitative trends on the effect of various parameters on the heat transfer occurring at droplet/wall interactions. Particularly, the effect of the initial surface temperature is observed to play a minor role, as long as it is low enough to prevent the occurrence of boiling. On the other hand, extreme wetting scenarios, such as superhydrophobicity limit the heat transfer between the spreading droplet and the surface. However, the thermal analysis reveals that a major reason for this is not related to the reduced contact time of the droplet on the surface (due to rebound) or air entrapment, but is rather associated to the reduced wetted area caused by the high contact angles.
\end{abstract}

\section{Keywords}

Droplet impact, heated surfaces, wettability, superhydrophobicity, Infrared high-speed thermography.

\section{Introduction}

Transport phenomena occurring at liquid-solid interfaces run a wide range of processes in various numerous applications. For instance, cooling systems are vital in different applications and at diverse temporal and spatial scales, such as electronics cooling, nuclear and chemical reactors, refrigeration systems, thermal generation of electricity or even in the food industry. Focusing on electronics cooling, the thermal management of microprocessors is argued to be the largest limitation to the development of new processors in the near future, which demands for innovative cooling strategies. Popular strategies include liquid cooling with phase change, such as pool boiling and spray cooling and often address surface modification to enhance the heat transfer processes, e.g. [1-2]. In any of these cases, the heat transfer is mostly governed by the mechanisms occurring at the liquid-(modified) surface interface. Despite being studied for many years, an accurate description of these processes is not provided yet, as it demands for experimental data obtained under demanding conditions, with high spatial and temporal resolutions. A clear example of these limitations is the study of droplet wall/interactions. Despite being studied for more than a century by many researchers as reviewed for instance in [3-4], the complex relation between droplet dynamics and heat transfer is far to be understood. The current knowledge on droplet/wall interactions recognizes wettability as playing a fundamental role in droplet dynamics and heat transfer processes. However, the accurate description of its effect is also still far from being completely described. Particularly, rudimentary knowledge is still achieved when complex surfaces with modified topography and/or chemistry are used [5]. This knowledge is even sparser when the complexity of the surfaces includes hierarchical structures, such as those present in biomimetic surfaces, which requires demanding static and dynamic wetting characterization methodologies, as recently reported by [6]. Infrared (IR) thermography has been recently explored as a high potential alternative to the intrusive measuring methods often used, based on thermocouples, which can provide important information on droplet impact under such particular boundary conditions [7-9]. However, many of these studies are focused on sessile droplets [10] or in particular boiling regimes such as the film boiling [11] where wettability plays a secondary role. Furthermore, care must be taken in the calibration and post-processing methods to obtain reliable measurements. Within this scope, the present paper explores the use of time resolved infrared thermography with high spatial resolution, combined with high-speed imaging to describe 
the droplet-surface interfacial heat transfer phenomena occurring during droplet impact onto heated surfaces. Custom made calibration and post-processing methods are proposed and discussed. The experimental impact conditions consider the effect of different parameters such as impact velocity and fluid properties. Emphasis is given to the effect of wettability (hydrophilic vs superhydrophobic surfaces).

\section{Material and methods}

Experimental set-up and working conditions

Droplets are generated at the tip of a hypodermic needle and fall by action of gravity on the heated surface. Water and ethanol are chosen as working fluids to address the effect of surface tension (partial wetting vs well wetting fluid) on the heat transfer processes occurring at the liquid-surface interface. The main physico-chemical properties of the working fluids is summarized in Table 1.

Table 1. Thermo-physical properties of the fluids used in the present work.

\begin{tabular}{l|cc}
\hline \hline Properties & Water & Ethanol \\
\hline \hline Saturation Temperature $-\mathrm{T}_{\text {sat }}\left[{ }^{\circ} \mathrm{C}\right]$ & 100 & 78.3 \\
Density $-\rho\left[\mathrm{kg} / \mathrm{m}^{3}\right]$ & 1000 & 757 \\
Dynamic viscosity $-\mu \times 10^{-3}\left[\mathrm{Ns} / \mathrm{m}^{2}\right]$ & 1.05 & 1.19 \\
Surface tension $-\sigma \times 10^{-3}[10-3 \mathrm{~N} / \mathrm{m}]$ & 72.88 & 22.8
\end{tabular}

Droplet initial diameters $D_{0}$ range between $2.4 \mathrm{~mm}<D_{0}<3 \mathrm{~mm} \pm 0.2 \mathrm{~mm}$ and the impact velocities $V_{0}$ are varied between $0.8 \mathrm{~m} / \mathrm{s}$ and $2 \mathrm{~m} / \mathrm{s}$. The impact surface is a stainless steel electrically heated foil, with $20 \mu \mathrm{m}$ thick, $20 \mathrm{~mm}$ wide and $100 \mathrm{~mm}$ long. The heating assembly consists in copper electrodes clamped on the top of the stainless steel foil, which is then glued on the top of an insulating thermal glass. This entire assembly is then placed on a stainless steel support for an easier positioning. The bottom side of the stainless steel foil used for IR thermography is black matt painted to increase the emissivity $(\varepsilon r=0.95)$. The surface is considered to be smooth, even after application of a chemical coating to turn it superhdrophobic. (variations in the average and in the peakto-valley roughness, measured with a profilemeter Dektak 3 from Veeco are smaller than 20nm). This commercial coating called Glaco® is mainly a perfluoroalkyltrichlorosilane combined with perfluoropolyether carboxylic acid and a fluorinated solvent.

Wettability is characterized measuring the quasi-static advancing and receding and the static contact angles, using an optical tensiometer (THETA from Attention). The static contact angle, measured by the sessile drop

method was $\theta=81.7^{\circ} \pm 1^{\circ}$ for the uncoated surface and $\theta=162.5^{\circ}$ for the coated surface. The quasi-static advancing and receding angles were used to evaluate the hysteresis of the foil (i.e. the difference between the quasi-static angles), which was always larger than $20^{\circ} \pm 1^{\circ}$ for the hydrophilic foils tested here and always lower than $10^{\circ}$ for the coated superhydrophobic surfaces. A detailed description of the experimental procedure taken to characterize the wettability of the surfaces can be found in [6].

The topography and wettability of each foil are carefully evaluated before and after droplet impact to assure consistent boundary conditions for each impact event. Furthermore, care was taken to assure that the initial surface temperature was reproducible before each new droplet impact.

An infrared IR-high speed camera (ONCA-MWIR-InSb from Xenics - ONCA 4696 series) is placed below the heated target, while a high-speed camera (Phantom v4.2) is mounted to take side views of the droplets. During impact, simultaneously but not synchronized high-speed video and high-speed thermographic images are taken to record the dynamic behavior of the droplets. The acquisition frequency and resolution are $2200 \mathrm{fps}$, $512 \times 512 \mathrm{px}^{2}$ and $1000 \mathrm{fps}$ and $150 \times 150 \mathrm{px}^{2}$ for the high-speed video and high-speed thermographic camera, respectively. For each experimental condition considered here, five tests were performed to assure reproducibility of the experiments.

\section{Post-processing methods}

Image post-processing is used to obtain the initial diameter $D_{0}$, the impact velocity $V_{0}$, the spreading diameter $D(t)$ and the spreading factor $\beta=D(t) / D_{0}$. The curves are averaged from at least 3 events taken at similar conditions.

The radial temperature profiles were obtained after post processing the IR images using a homemade MatLab code which allowed converting the raw IR images to temperature data. For the calibration method, a custom- 
made cavity based blackbody radiator device was designed and assembled. For each imposed temperature at the cavity and after achieving a stable condition for which the temperature is homogeneous in the cavity, the measured temperatures are converted into radiated energy flux $\left(\mathrm{W} / \mathrm{m}^{2}\right)$ performing an energy balance and plotted against the received intensity signal in ADU's (Analogic to Digital Units). Then a polynomial curve is fitted to these data. This calibration is performed considering a pixel by pixel approach. As for the post-processing procedure, a number of steps must be performed and a sequence of filters was developed and applied, as follows:

\section{1) Background removal filter}

To remove background noise from the the image, obtaining real temperature variations requires removing the image background. While a common background filter removes the background (the grey scale value) leaving visible changes in the following frames, the custom-made filter designed here considers the temperature variations to be relative to the background temperature. The developed filter is applied to each pixel as given in equation 1:

$\operatorname{vid}^{*}\left(x_{p}, y_{p}, t_{n}\right)=\left(\frac{\operatorname{vid}\left(x_{p}, y_{p}, t_{n}\right)-v i d\left(x_{p}, y_{p}, 1\right)}{\operatorname{vid}\left(x_{p}, y_{p}, 1\right)}\right) \operatorname{avTemp}+\operatorname{avTemp}$

where vid is a $3 d$ matrix with the temperatures for each pixel and for different timesteps, vid* is the resulting matrix, $x_{p}$ and $y_{p}$ are the Cartesian coordinates for the pixel, $t_{n}$ the timestep and avTemp is the average background temperature.

\section{2) Noise removal filter}

A median filter was used to perform noise removal. This filter has the potential to remove random bad pixel noise from the picture. It uses a MATLAB function that outputs the median of a 3-by-3 neighbourhood of the input pixel.

\section{Measurement uncertainties}

The main uncertainties associated to droplet dynamics are summarized in Table 2, while Table 3 depicts the uncertainties associated to the main parameters used to describe the heat transfer process at droplet/wall interaction.

Table 2. Uncertainties of the main parameters used to describe droplet dynamics.

\begin{tabular}{l|l}
\hline \hline Parameter & Uncertainties (rel. or abs) \\
\hline $\begin{array}{l}\text { Droplet diameter before impact } D_{o} \\
{[\mathrm{~mm}]} \\
\text { Spreading diameter } D[\mathrm{~mm}]\end{array}$ & $U_{D}= \pm 160 \mu \mathrm{m}$ \\
& $U_{D_{o}}= \pm 160 \mu \mathrm{m}$ \\
Spreading factor $\beta=D / D_{o}[-]$ & $u_{D^{\prime} D_{o}} \max = \pm 37 \%$ at $\left(D / D_{o}=0.17\right)$ \\
& $u D / D_{o} \operatorname{mim}= \pm 7 \%$ at $\left(D / D_{o}=3.86\right)$ \\
Impact velocity $V_{0}[\mathrm{~m} / \mathrm{s}]$ & $U_{V_{o}}=0.08[\mathrm{~m} / \mathrm{s}]$
\end{tabular}

Table 3. Uncertainties of the main parameters used to describe the heat transfer process during droplet spreading.

\begin{tabular}{l|l}
\hline \hline Parameter & Uncertainties U (rel. or abs) \\
\hline \hline Temperature $\mathrm{T}[\mathrm{K}]$ & $U_{T}= \pm 1 \mathrm{~K}$ \\
Temperature difference $\Delta \mathrm{T}[\mathrm{K}]$ & $U_{\Delta T}= \pm 1.4 \mathrm{~K}$ \\
& $u_{\Delta T} \max = \pm 14 \%$ at $(\Delta T=10 \mathrm{~K})$ \\
& $u_{\Delta T} \min = \pm 1.7 \%$ at $(\Delta T=78 \mathrm{~K})$ \\
Non-dimensional temperature $T^{*}[-]$ & $U_{T^{*} \max = \pm 0.04 \text { at }\left(T^{*}=1\right)}$
\end{tabular}


Imposed volumetric heat flux $q^{\prime \prime \prime}\left[W / m^{3}\right]$

Radial distance $r[\mathrm{~mm}]$

$$
\begin{aligned}
& U_{T^{*}} \min = \pm 0.02 \text { at }\left(T^{*}=0.3\right) \\
& u_{T^{*}} \max = \pm 6 \% \text { at }\left(T^{*}=0.3\right) \\
& u_{T^{*}} \max = \pm 4 \% \text { at }\left(T^{*}=1\right) \\
& u q^{\prime \prime \prime} \max = \pm 12 \% \text { at }\left(q^{\prime \prime \prime}=6.5 \cdot 10^{6}\left[\mathrm{~W} / \mathrm{m}^{3}\right]\right) \\
& U_{r}= \pm 200 \mu m
\end{aligned}
$$

\section{Results and discussion}

Simultaneous analysis of droplet dynamics and thermal processes

Although the IR and the high-speed cameras were not perfectly synchronized, the simultaneous images captured during droplet impact and spreading allow understanding the qualitative relation between droplet dynamics and the heat transfer processes occurring during the spreading. Hence, Figure 1 depicts the impact of a water droplet $\left(D_{0}=2.6 \mathrm{~mm}, V_{0}=0.8 \mathrm{~m} / \mathrm{s}\right)$ on the smooth uncoated (i.e. hydrophilic) stainless steel foil, initially heated at $T_{w 0}=80^{\circ} \mathrm{C}$. The figure shows the side view of the impacting droplet and the corresponding thermal images taken on the heated foil (bottom view) during impact and spreading. The corresponding temperature profiles, from the center of the droplet $(r=0 \mathrm{~mm})$ to the rim of the lamella are shown at the bottom images. The figure clearly shows the sudden temperature decrease at $r=0 \mathrm{~mm}$, at the point of impact, which quickly recovers to the rim, at the early instants after impact. A steeper temperature difference between the center of the droplet and the rim is then observed at later instants after impact, near the maximum spreading ( $t=6 m s)$, which remains for later stages, during recoiling, for the wetted area, while heat is removed at the liquid-solid interface. The rim is perfectly identified in the thermal images, which can be related to the temperature profiles, by the slight bump, associated to the change in the thickness of the lamella.

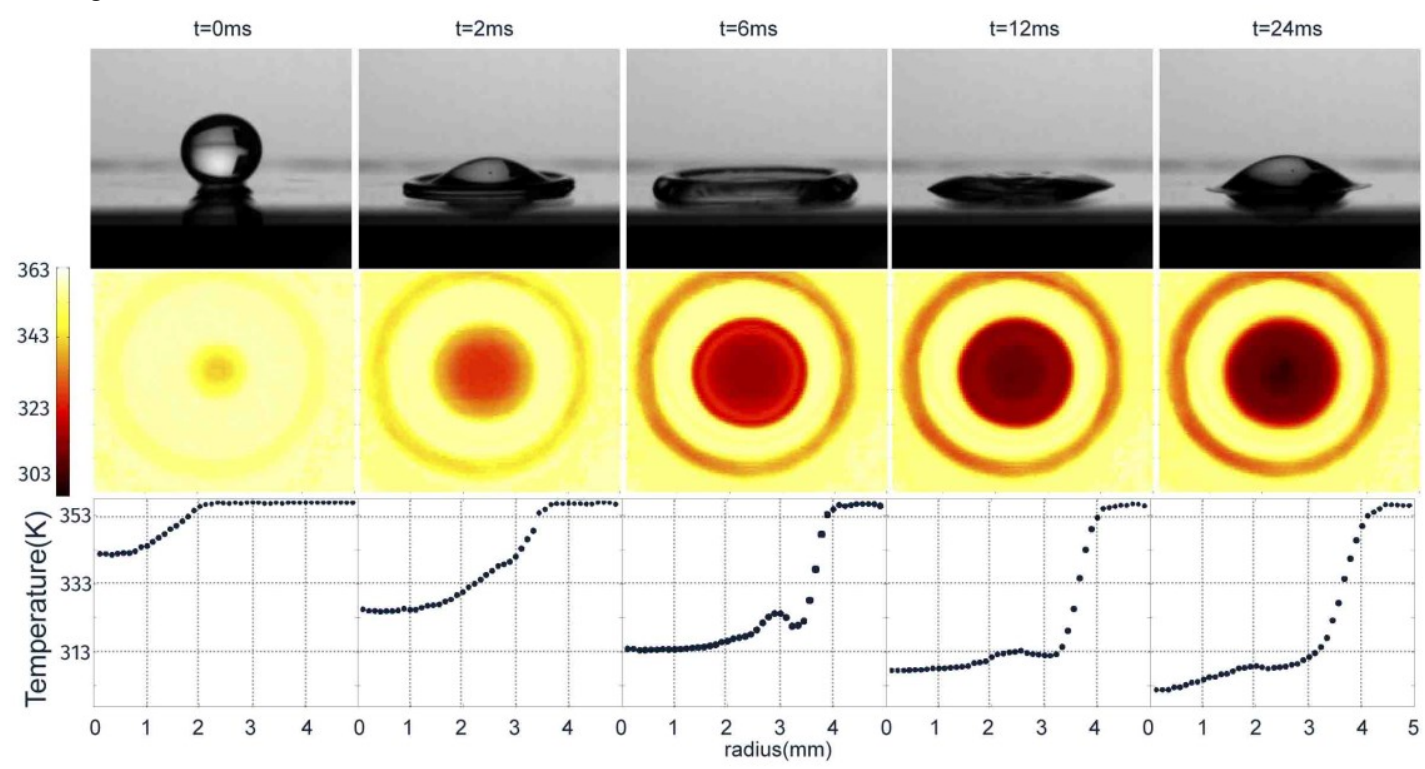

Figure 1. Correspondence between the high-speed images taken to the droplet (dynamic behaviour) and the IR thermal images taken to the surface in contact with the spreading droplet (heat transfer process) and to the resulting temperature profiles taken at various instants after impact. The water droplet $D_{0}=2.6 \mathrm{~mm}$ impacts the smooth stainless steel surface at $V_{0}=0.8 \mathrm{~m} / \mathrm{s}$. Initial surface temperature $T_{w 0}=80^{\circ} \mathrm{C}$.

\section{Evaluating the calibration method}

To infer on the efficacy of our calibration method, some preliminary images were taken using the camera's default calibration, which were then compared to those taken using the custom-made calibration. For illustrative purposes the result of applying our calibration process is discussed for the impact of water droplets at $2 \mathrm{~m} / \mathrm{s}$ and $0.8 \mathrm{~m} / \mathrm{s}$, for initial foil temperatures of $60^{\circ} \mathrm{C}, 100^{\circ} \mathrm{C}$ and $110^{\circ} \mathrm{C}$. Real working temperature values is provided, so small variations can be observed around these reference values. 
Figure 2 depicts the temperature variation on the foil, as a function of the spreading radius, of a water droplet $\left(D_{0}=2.6 \mathrm{~mm}\right)$ impacting on the smooth and hydrophilic stainless steel foil at $V_{0}=0.8 \mathrm{~m} / \mathrm{s}$ at the initial foil temperature $T_{w 0}=107^{\circ} \mathrm{C}$ (Figure 2a) and $T_{w_{0}}=117^{\circ} \mathrm{C}$ (Figure 2b). Particularly, this Figure compares the results obtained using the camera's default calibration (Figure 2a) with those attained after applying our calibration and post-processing method (Figure $2 b$ ). The different curves in each plot correspond to different time instants after impact. Hence, at the time instant immediately after impact $($ e.g. $t=1 \mathrm{~ms})$ the temperature decrease observed on the impact region of the foil (central region of the droplet) due to the contact with the cold liquid is small and swiftly recovers for the small spreading factor that is still observed at that instant. This trend contrasts with the higher temperature drops at later time instants after impact. Despite the small variations that occurred in the initial impact conditions, which could not be entirely controlled, the Figure clearly shows that during the spreading phase (all the points before $t=13 \mathrm{~ms}$ ), the calibration and post-processing method proposed here are able of capturing the effect of air entrapment air in the stagnation point (the point of droplet impact) often referred in the literature, e.g. [7], which is related to the small temperature decrease that is observed from the point of impact $(r=0 \mathrm{~mm})$ to the immediately subsequent points.

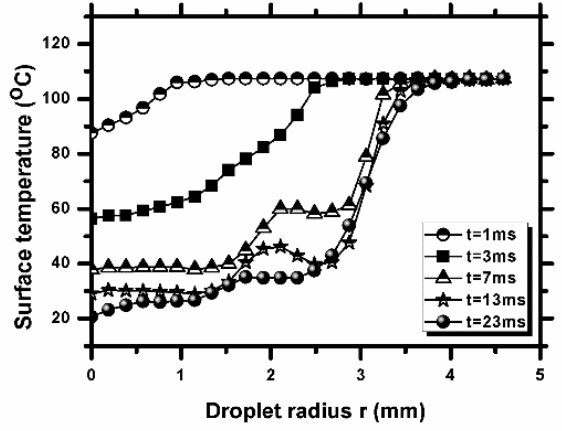

a)

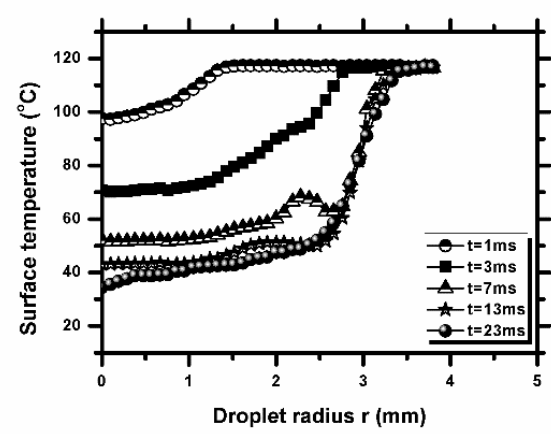

b)

Figure 2. Temperature variation on the stainless steel foil (smooth and hydrophilic, with an equilibrium angle $\theta=87.1^{\circ} \mathrm{I}$ ) during impact and spreading of a water droplet $\left(D_{0}=3 \mathrm{~mm}, U_{0}=0.8 \mathrm{~m} / \mathrm{s}\right)$, for an initial foil temperature of: a) $T_{w 0}=107^{\circ} \mathrm{C}$. The results were obtained from the thermal images after applying the camera's default calibration. These results were taken for an ambient temperature $T_{a m b}=21^{\circ} \mathrm{C}$; b) $T_{w o}=117^{\circ} \mathrm{C}$. The results were obtained after applying our custom-made calibration and postprocessing method. The ambient temperature in this case was $T_{a m b}=24^{\circ} \mathrm{C}$.

The calibration and post-processing procedures proposed here also allowed amending some physically incorrect results that were often obtained with the camera's default calibration. For instance, in Figure 2a, for the curve obtained at $t=23 \mathrm{~ms}$ after impact, the foil temperature at the impact region $(r=0 \mathrm{~mm})$ is slightly lower than the ambient temperature, which is not possible, being corrected in our custom-made method.

As the temperature profiles alone are not enough to characterize the heat transfer process, further analysis can be performed by computing the heat flux and the so-called cooling effectiveness $\varepsilon$, as defined by [7]. Considering an axisymmetry condition at the center of the droplet, the heat flux removed by the droplet from the foil $q$ " can be written as:

$q^{\prime \prime}=q_{0}^{\prime \prime}+k_{\delta} \frac{\partial^{2} T}{\partial r^{2}}-\rho C_{p} \delta \frac{\delta T}{\delta t}$

Here, $k_{\delta}, \rho, C_{p}$ and $\delta$ are the thermal conductivity, density, heat capacity and thickness of the heated foil, respectively. Assuming that there is no phase change of the liquid during droplet impact and spreading, [7] define as cooling efficiency $\varepsilon$, the ratio between the actual sensitive heat removed by the droplet and the maximum sensitive heat that can be theoretically removed:

$\varepsilon=\frac{\int_{t} \int_{A} q^{\prime \prime} d A d t}{\left(m C_{p} \Delta T\right)_{\text {water }}}$

These quantities were used to evaluate the effect of various parameters in droplet dynamics and heat transfer. The results were analysed to describe the heat transfer processes coupled with the fluid flow and dynamic characteristics during droplet impact.

Effect of the initial surface temperature $T_{\text {wo }}$

Although the detailed results cannot be shown here, due to paper length constrains, the analysis of the temperature profiles, the heat fluxes and the cooling effectiveness shows a negligible effect of the initial surface temperature, as all the curves obtained at different initial surface temperatures tend to collapse. Exception was 
made to the curve obtained at $T_{w 0}=110^{\circ} \mathrm{C}$, which tends to converge for higher values of the cooling effectiveness, which may be attributed to the beginning of the liquid phase change and to an incipient boiling.

\section{Effect of liquid surface tension}

To infer on how good our method captures the physics governing the fluid dynamics and heat transfer processes at droplet/wall interactions, the effect of the liquid surface tension is addressed here comparing the thermal processes occurring at the impact of water and ethanol droplets for initial surface temperatures below saturation. Given the lower surface tension of ethanol, the spreading factor is much larger than that of water, so the lamella is also much thinner. Furthermore, after spreading, good wetting fluids like ethanol tend to proceed the spreading for very long time intervals after impact, without recoiling, in a regime controlled by capillary forces [12-13], often aided by the formation of a precursor film [12]. This reduced thickness of the lamella of the ethanol droplet became an obstacle when performing the experiments, since for initial foil temperatures above saturation, the applied electrical current is very high deforming the stainless-steel foil. These deformations are not relevant for the spreading of the water droplets but are enough to cause the ethanol lamella to slip away from the measurement area, being impossible to capture IR images under those conditions. Hence, measurements could be performed for ethanol only at the lowest impact velocity $\left(V_{0}=0.8 \mathrm{~m} / \mathrm{s}\right)$ and for initial foil temperatures of $40^{\circ} \mathrm{C}$ and $60^{\circ} \mathrm{C}$. The spreading factor $\beta=D(t) / D_{0}$ obtained for water and ethanol droplets is depicted in Figure 3 . The maximum spreading factor is reached at $7 \mathrm{~ms}$ after impact for ethanol and $5 \mathrm{~ms}$ after impact for water. Naturally, the wetted area of the spreading ethanol droplet is larger than that of water.

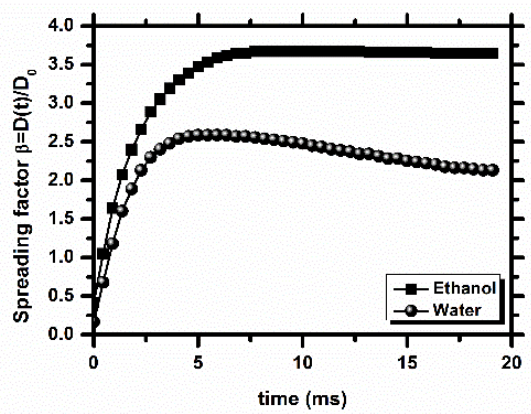

Figure 3. Temporal evolution of the spreading factor for a water and an ethanol droplet impacting on the smooth and uncoated hydrophilic stainless steel surface at $V_{0}=0.8 \mathrm{~m} / \mathrm{s}$. $T_{w 0}=60^{\circ} \mathrm{C}$.

Comparing the heat flux removed by the water and the ethanol droplet, as depicted in Figure 4, the heat flux peak is more accentuated for the water droplet. This is due to the the thicker lamella edge, caused by a higher surface tension and naturally to the higher values of the thermal properties of the water. So, overall the heat flux removed by the water is about twice that removed by the ethanol. The fact that the temperature difference to the saturation temperature is different in both liquids seems not to have a significant effect in this case. The radius is made nondimensional since the initial diameter of the ethanol droplets is slightly smaller than that of the water droplets.

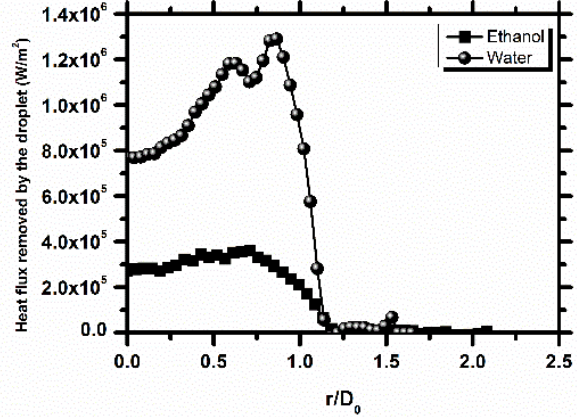

a) $t=2 m s$ after impact

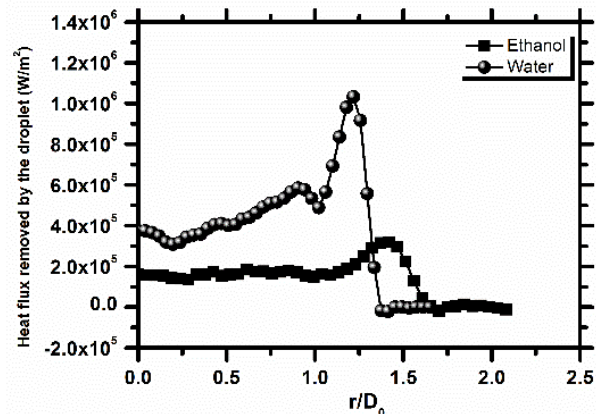

b) $\mathrm{t}=4 \mathrm{~ms}$ after impact

Figure 4. Heat flux along the radius for or a water and an ethanol droplet impacting on the smooth and uncoated hydrophilic stainless steel surface at $V_{0}=0.8 \mathrm{~m} / \mathrm{s}$. $T_{w_{0}}=60^{\circ} \mathrm{C}$. 
Although the heat flux is difficult to compare between the water and the ethanol droplets, the cooling effectiveness can be easily compared for both liquids, as it considers the effect of both the droplet's initial diameter and thermal properties. The cooling effectiveness computed as a function of the non-dimensional time $t^{*}=t V_{0} / D_{0}$, for the ethanol and water droplets is depicted in Figure 5, which clearly shows a higher cooling effectiveness for ethanol.

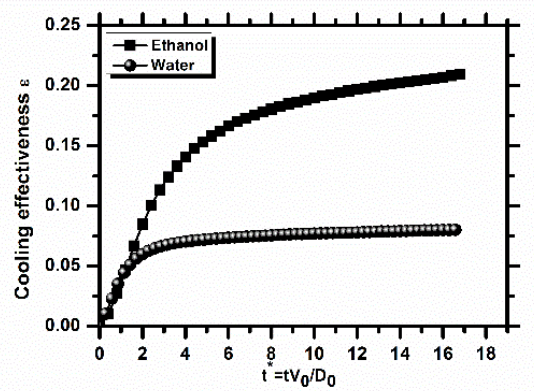

Figure 5. Comparison between the cooling effectiveness of a water and an ethanol droplet impacting on the smooth and uncoated hydrophilic) stainless steel surface at $V_{0}=0.8 \mathrm{~m} / \mathrm{s}$. $T_{w 0}=60^{\circ} \mathrm{C}$.

This result may seem odd, following the previous analysis of the heat flux. Nevertheless, considering the definition of the cooling effectiveness (the total heat removed is divided by the maximum possible heat removed) this result is actually plausible. Since ethanol has worse thermal properties, the maximum possible heat removed is also lower than that of water, so in the end the cooling effectiveness considering the improved wetted area is actually higher for ethanol. It is worth noting, however, that this better cooling effectiveness is not associated to a better cooling performance, as one must evaluate all the parameters and effectively access the heat that is removed from the surface, which is naturally larger for water.

\section{Effect of surface wettability}

To infer on the effect of wettability, the heat flux (Figure 6) and cooling effectiveness (Figure 7) are compared for the impact of a water droplet $\left(D_{0}=2.6 \mathrm{~mm}, V_{0}=0.8 \mathrm{~m} / \mathrm{s}\right)$ on the stainless steel foil, with and without the Glaco $尺$ coating. As aforementioned, the coated foil becomes superhydrophobic, depicting equilibrium angles with water of $\theta=162.5^{\circ}$ with a hysteresis always lower than $10^{\circ}$. Although the spreading factor is similar for both droplets, the shape of the rim is quite different. Hence, while in the hydrophilic case, the droplet spreads and wets the surface, in the superhydrophobic case, the shape of the rim reduces the true wetted area, thus reducing the liquid-solid contact area. The superhydrophobic surface also promotes the complete rebound of the droplet, which occurs approximately at $t=20 \mathrm{~ms}$ after impact. Consequently, the heat flux depicted in Figure 6 is considerably smaller for the superhydrophobic surface. The heat flux peaks at the contact edge are also smaller. The peak observed at $t=4 \mathrm{~ms}$ is associated to the maximum spreading diameter which occurs at that time instant (Figure 6b).

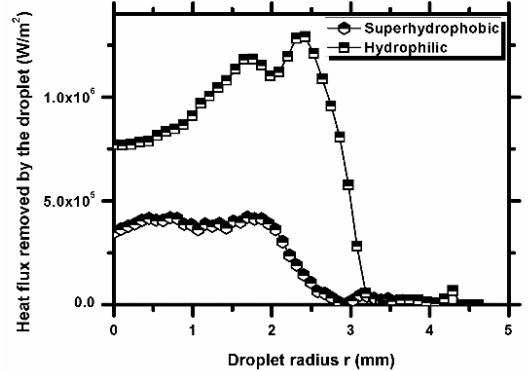

a) $t=2 m s$ after impact

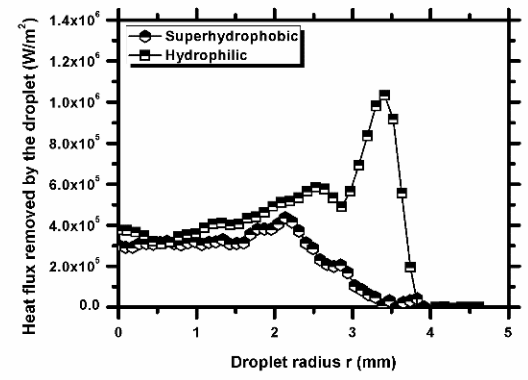

b) $t=4 m s$ after impact

Figure 6. Heat flux during the spreading of a water droplet on a hydrophilic vs superhydrophobic surface $\left(D_{0}=2.6 \mathrm{~mm}\right.$, $V_{0}=0.8 \mathrm{~m} / \mathrm{s}, T_{w_{0}}=100^{\circ} \mathrm{C}$ ).

The lower cooling effectiveness computed as a function of the non-dimensional time $t^{*}=t V_{0} / D_{0}$ for the superhydrophobic surface is therefore not only related to the reduced contact time between the droplet and the surface due to droplet rebound, but also due to the reduced wetting contact area resulting from the high contact angles. Despite the spatial resolution does not allow the clear detection of very thin layers of air, it is good enough to detect bubble entrapment, as discussed in Figure 1. From this perspective, the presence of an air layer precluding droplet contact with the superhydrophobic surface is not evident, except at the contact line edges 
where an important part of the heat transfer takes part. Hence, the reduced contact area due to the shape of the droplet visualization seems to play a major role in the heat transfer process, although a more detailed analysis and relative quantification is required in the near future to confirm these trends.

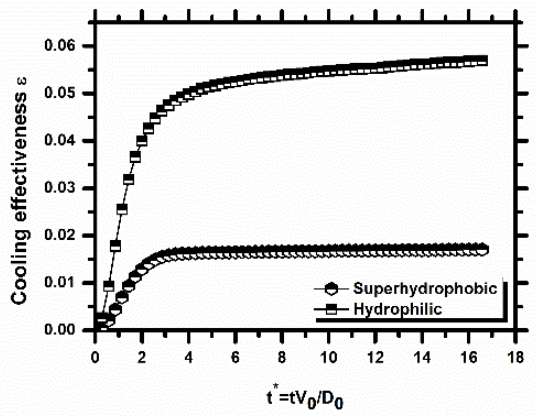

Figure 7. Cooling effectiveness as a function of the non-dimensional time $t^{*}$, for a water droplet spreading on a hydrophilic vs superhydrophobic surface $\left(D_{0}=2.6 \mathrm{~mm}, V_{0}=0.8 \mathrm{~m} / \mathrm{s}, T_{w_{0}}=100^{\circ} \mathrm{C}\right)$.

\section{Conclusions}

The present paper analyses the liquid-surface interfacial heat transfer phenomena occurring at droplet/wall interactions combining the use of time resolved infrared thermography with high-speed imaging. The results show that the methodology proposed captures well particular details on droplet dynamics and heat transfer, allowing to identify air bubble trapping at the impact region as well as the temperature variations at the formation of the rim. The combined analysis of droplet dynamics (e.g. the spreading factor) with the radial temperature profiles, heat flux and cooling effectiveness computation allowed establishing qualitative and quantitative trends on the effect of various parameters on the heat transfer occurring at droplet/wall interactions. Hence, the effect of the initial surface temperature is observed to play a minor role, as long as it is low enough to prevent the occurrence of boiling. The physico-chemical properties of the working fluids naturally play a vital role in the heat transfer process, so the heat fluxes obtained during the spreading of a water droplet are much larger than those obtained during the spreading of an ethanol droplet (up to two times higher). However, the cooling effectiveness of the ethanol droplet is actually higher than that of the water droplet given its larger spreading area, which is accounted in the effectiveness, together with the properties of the fluids. The superhydrophobic wetting regime limits the heat transfer between the spreading droplet and the surface, as expected, given the particular dynamic behaviour of the droplets within this regime (limited droplet-surface contact due to air entrapment and occurrence of rebound). However, the thermal analysis reveals that a major reason for this is not related to the reduced contact time of the droplet on the surface (due to rebound) or air entrapment, but is rather associated to the reduced wetted area caused by the high contact angles.

\section{Acknowledgements}

The authors are grateful to Fundação para a Ciência e Tecnologia (FCT) for partially financing the research under the framework of the project RECI/EMS-SIS/0147/2012 and for supporting P. Pontes with a research fellowship. A. S. Moita acknowledges FCT for financing her contract through the IF 2015 recruitment program (IF 008102015) and E. acknowledges FCT for supporting his PhD fellowship (SFRH/BD/88102/2012).

\section{References}

[1] Panão, M., Guerreiro, J. and Moreira, A.L.N., 2012, Int. J. Heat Mass Transf. 55, pp. 2854-2863.

[2] Moura, M., Teodori, E., Moita, A.S. and Moreira, A.L.N., 2016, Proc. IEEE ITherm Conference, The Intersociety Conference on Thermal and Thermomechanical Phenomena in Electronic Systems, pp. 378-387.

[3] Moreira, A.L.N., Moita, A.S. and Panão, M.R., 2010, Prog Energy Comb Sci., 36, pp. 554-580.

[4] Liang, G., and Mudawar, I., 2017, Int. J. Heat Mass Transf., 106, pp. 103-126.

[5] Marengo, M., Antonini, C., Roisman, I.V. and Tropea, C., 2011, Curr. Op. Coll. Interface Sci., 16, pp. $292-302$.

[6] Pereira P., Moita, A. S., Monteiro, G. and Prazeres, D.M.F., 2014, J. Bionic Eng., 11(3), pp. 346-359.

[7] Pasandideh-Fard, M., Aziz, S., Chandra, S. and Mostaghimi, J., 2001, Int. J. Heat Fluid Flow, 22, pp. 201-210.

[8]Girard, F., Antoni, M. and Sefiane, K. 2010, Langmuir, 26, pp. 4576-4580.

[9] Kim, H. and Bungiorno, J., 2011, Int. J. Multiphase Flow, 37, pp. 166-172.

[10] Tartarini, P., Corticelli, M.A. and Tarozzi, L., 2009, Appl. Thermal Eng., 29, pp. 1391-1397.

[11] Gradeck, M., Seiler, N., Ruyer, P. and Maillet, D., 2013, Exp. Thermal Fluid Sci., 47, pp. 14-25.

[12] Moita, A.S. and Moreira, A.L.N., 2003, $9^{\text {th }}$ ICLASS 2003, Sorrento, Italy.

[13] Rioboo, R., Marengo. M. and Tropea, C., 2002, Exp. Fluids, 33(1), pp. 112-124. 